\title{
Recent advances in preimplantation genetic diagnosis
}

\author{
KY Leung*, MD, FHKAM (Obstetrics and Gynaecology) \\ Department of Obstetrics and Gynaecology, Queen Elizabeth Hospital, Jordan, Hong Kong
}

Hong Kong Med J 2015;21:296-7

*leungky1@ha.org.hk

DOI: 10.12809/hkmj154638

Preimplantation genetic diagnosis (PGD) gives couples who are at risk of having a child with an inherited genetic disorder or chromosome abnormality, a chance to have an unaffected child without undergoing termination or miscarriage of an affected pregnancy. Embryos obtained from in-vitro fertilisation (IVF) with or without intracytoplasmic sperm injection are tested genetically prior to selective transfer of unaffected ones into the uterus. The physical and psychological complications of a termination or miscarriage, especially in repeated situations, should not be underestimated.

In PGD, DNA can be obtained by blastomere biopsy at the cleavage stage, trophectoderm cell biopsy when an embryo has developed to the blastocyst stage or biopsy of one or both polar bodies. Compared with cleavage stage biopsy, trophectoderm biopsy does not adversely impact the embryo, although vitrification and cryopreservation of the embryo may be required to allow time for genetic analysis. ${ }^{1}$ Although polar body biopsy is less invasive, it is less predictive of actual clinical outcome than direct embryo assessment. ${ }^{2}$

Genetic laboratories have developed their own protocols to perform different molecular tests on the limited amount of DNA obtained from biopsy. Traditionally, fluorescent in-situ hybridisation is used for cytogenetic diagnosis, and polymerase chain reaction for molecular diagnosis. New technologies, including array comparative genomic hybridisation $(\mathrm{CGH})$ and single nucleotide polymorphism (SNP) microarrays, can improve diagnostic accuracy. The single-cell whole genome amplification (WGA) method allows subsequent mutation study, directly by minisequencing and/or indirectly by linkage analysis alongside the mutation test. It also allows simultaneous PGD for more than one indication. ${ }^{5}$

The indications for PGD are increasing. Common ones include single-gene disorders, X-linked diseases, and inherited chromosome abnormalities. Preimplantation genetic diagnosis of predisposition to inherited cancer such as breast cancer (BRCA mutation) is also emerging. ${ }^{6}$ Nonetheless, social sexing is prohibited in Hong Kong and Europe. Legislation and regulation of PGD also vary among different countries.

Aneuploidy is the most common cause of repeated implantation failure and recurrent miscarriage. As such, preimplantation genetic screening (PGS), using similar technology to PGD, is offered to improve delivery rates in patients of advanced maternal age, and in those with repeated implantation failure, repeated miscarriages, and severe male factor infertility. Evidence that PGS can help improve delivery rates is conflicting, however. ${ }^{7}$ Whether PGS using array CGH or SNP microarrays can increase delivery rates requires further study.

In 2006, a tertiary centre in London reported their experience of 330 PGD cycles including 96 cycles for single-gene disorders and 62 cycles for $\mathrm{X}$ linked disorders. ${ }^{8}$ In $62 \%$ of the cycles, there was at least one unaffected embryo available for transfer, resulting in 90 pregnancies, 68 clinical pregnancies, and 58 live births. The clinical pregnancy rate and live birth rate was $33 \%$ and $28 \%$ per cycle started, respectively. ${ }^{8}$ This result was similar to a clinical pregnancy rate of $30.2 \%$ per transfer reported by the European Society of Human Reproduction and Embryology PGD Consortium in 2014. ${ }^{9}$

In this issue, authors in a local tertiary centre reported their 6-year experience of 124 PGD cycles for monogenetic diseases in 76 couples using WGA and linkage analysis. ${ }^{10}$ The ongoing pregnancy rate was $28.2 \%$ per initiated cycle and $38.0 \%$ per fresh embryo transfer. ${ }^{10}$ These pregnancy rates were similar to those of PGD using frozen-thawed embryo transfer cycles and for IVF for routine infertility treatment. Approximately $19 \%$ of the cycles for PGD were cancelled after initiation of stimulation. Approximately $70 \%$ of PGD was performed for thalassaemia ( $\alpha$ or $\beta$ ), and the remaining $30 \%$ for 19 other monogenetic diseases that included spinocerebellar ataxia type 3 and Huntington's disease. No misdiagnosis was found in this small series according to the available data. ${ }^{10}$

In clinical practice, thalassaemia is the most common single-gene disorder in Hong Kong. When both parents are a $\beta$-thalassaemia carrier, there is a $25 \%$ risk of having a fetus affected by homozygous $\beta$-thalassaemia. Conventional prenatal diagnosis is an option but couples will face termination of pregnancy if an affected pregnancy is detected. For an unaffected pregnancy, human leukocyte antigen (HLA) typing can subsequently be performed but may not be compatible with a previously affected child. On the other hand, PGD allows at-risk couples 
the chance to have an unaffected child without undergoing termination or miscarriage of an affected pregnancy. In addition, PGD can be offered to at-risk couples even in the absence of a previously affected pregnancy. It can also allow selection of an unaffected and HLA-compatible embryo simultaneously before transfer into the uterus. This results in subsequent availability of HLA-matched cord blood at birth for transplant to an affected elder sibling.

In a local study of women at risk, ${ }^{11}$ in particular those with subfertility problems or with a child affected by major thalassaemia, PGD provided an acceptable alternative to conventional prenatal diagnosis. Couples also had a positive attitude to the use of PGD/HLA typing to reproduce a 'saviour child' to save an affected sibling. ${ }^{12}$ It is unknown, however, whether Hong Kong women at risk of other genetic disorders share this view.

Preimplantation genetic diagnosis requires close collaboration between different specialists including obstetricians, fertility specialists, IVF laboratory, and human geneticists. It needs intensive effort and expensive techniques, and is demanding for the patients. In Hong Kong, the high costs of PGD and IVF must be borne by the patient. It is important to inform patients about the success rate and potential risks of IVF and PGD, possible complications of ovarian hyperstimulation, and the risk of multiple pregnancy.

Because of the possibility of mosaicism related to blastomere or trophectoderm cell biopsy and the false-negative rate due to allelic dropout or contamination related to the limited amount of DNA obtained from PGD, prenatal diagnosis is still recommended after PGD. Prenatal diagnosis is made following an invasive test such as chorionic villus sampling or amniocentesis or, in suitable situations, by a non-invasive approach such as testing of cellfree fetal DNA in maternal plasma for chromosomal abnormalities or by serial ultrasound examinations to exclude haemoglobin Bart's disease.

There are ethical concerns that arise with new technologies such as microarrays and WGA that generate more detailed and complex genetic information than previous conventional approaches, and make preconception carrier testing feasible. ${ }^{13}$ Genetic laboratories should report their results according to internationally accepted accreditation standards. ${ }^{14}$ Adequate pre-testing counselling is important.

With a multidisciplinary approach and advances in technology, PGD is a great opportunity for couples at risk. It allows them to have an unaffected child while avoiding the need to terminate an affected pregnancy, and makes HLA-matched cord blood available at birth for transplantation to a previously affected child. Nonetheless, the process is expensive, demanding for couples, not always successful, and not without risks. Couples at risk should be well informed about the two reproductive options, namely PGD and prenatal diagnosis, in prepregnancy counselling.

\section{References}

1. Zhang S, Tan K, Gong F, et al. Blastocysts can be rebiopsied for preimplantation genetic diagnosis and screening. Fertil Steril 2014;102:1641-5.

2. Salvaggio CN, Forman EJ, Garnsey HM, Treff NR, Scott RT Jr. Polar body based aneuploidy screening is poorly predictive of embryo ploidy and reproductive potential. J Assist Reprod Genet 2014;31:1221-6.

3. Rubio C, Rodrigo L, Mir P, et al. Use of array comparative genomic hybridization (array-CGH) for embryo assessment: clinical results. Fertil Steril 2013;99:1044-8.

4. Tobler KJ, Brezina PR, Benner AT, Du L, Xu X, Kearns WG. Two different microarray technologies for preimplantation genetic diagnosis and screening, due to reciprocal translocation imbalances, demonstrate equivalent euploidy and clinical pregnancy rates. J Assist Reprod Genet 2014;31:843-50.

5. Vendrell X, Bautista-Llácer R. A methodological overview on molecular preimplantation genetic diagnosis and screening: a genomic future? Syst Biol Reprod Med 2012;58:289-300.

6. Derks-Smeets IA, Gietel-Habets JJ, Tibben A, et al. Decision-making on preimplantation genetic diagnosis and prenatal diagnosis: a challenge for couples with hereditary breast and ovarian cancer. Hum Reprod 2014;29:1103-12.

7. Mastenbroek S, Twisk M, van der Veen F, Repping S. Preimplantation genetic screening: a systematic review and meta-analysis of RCTs. Hum Reprod Update 2011;17:45466.

8. Grace J, El-Toukhy T, Scriven P, et al. Three hundred and thirty cycles of preimplantation genetic diagnosis for serious genetic disease: clinical considerations affecting outcome. BJOG 2006;113:1393-401.

9. Moutou C, Goossens V, Coonen E, et al. ESHRE PGD Consortium data collection XII: cycles from January to December 2009 with pregnancy follow-up to October 2010. Hum Reprod 2014;29:880-903.

10. Chow JF, Yeung WS, Lee VC, Lau EY, Ho PC, Ng EH. Experience of more than 100 preimplantation genetic diagnosis cycles for monogenetic diseases using wholegenome amplification and linkage analysis in a single centre. Hong Kong Med J 2015;21:299-303.

11. Hui PW, Lam YH, Chen $M$, et al. Attitude of at-risk subjects towards preimplantation genetic diagnosis of alpha- and beta-thalassaemias in Hong Kong. Prenat Diagn 2002;22:508-11.

12. Hui EC, Chan C, Liu A, Chow K. Attitudes of Chinese couples in Hong Kong regarding using preimplantation genetic diagnosis (PGD) and human leukocyte antigens (HLA) typing to conceive a 'Saviour Child'. Prenat Diagn 2009;29:593-605.

13. Hens K, Dondorp W, Handyside AH, et al. Dynamics and ethics of comprehensive preimplantation genetic testing: a review of the challenges. Hum Reprod Update 2013;19:366-75.

14. Harper J, Geraedts J, Borry P, et al. Current issues in medically assisted reproduction and genetics in Europe: research, clinical practice, ethics, legal issues and policy. Hum Reprod 2014;29:1603-9. 